\title{
CONSTRUCTION TECHNOLOGY OF PERMEABLE RIB DOUBLE-ARCH TUNNEL
}

\author{
Ruijun Song ${ }^{1, a}$, Fei Yu ${ }^{1, b}$, Shanxiong Chen ${ }^{1}$, Xiuhe Chen ${ }^{2}$, Fei Wang ${ }^{2}$ \\ ${ }^{1}$ Institute of Rock and Soil Mechanics, Chinese Academy of Sciences, Wuhan, 430071, China \\ ${ }^{2}$ Anhui Communications Planning, Design and Research Institute, Hefei, 230041, China \\ a398449801@qq.com, byufei8720@163.com
}

Keywords: permeable rib double-arch tunnel, tunnel adjacent to mountain, construction method; bias voltage, surrounding rock consolidation

\begin{abstract}
The permeable rib double-arch tunnel is a new type structure of tunnel adjacent to mountain, a better way to solve the problems of engineering stability and environmental protection of highway adjacent to mountain in mountains area. For the environmental geological problems of the tunnel adjacent to mountain with the thin layer and the significant biasing effect, a set of more suitable construction methods and techniques are put forward combining with the structural feature and the analysis of mechanics regularity to the special-shaped tunnel. With practical engineering, the construction technology of the permeable rib double-arch tunnel has a better effectiveness and reliability, which ensures the successful implementation of the new type of tunnel structure.
\end{abstract}

\section{Introduction}

The route trend of highway adjacent to mountain is parallel or inclined to the hillside, which ever uses the scheme of deep excavation cut and it not only causes serious damage to the surrounding vegetation, but also brings stability problems of the high cut slope. At present, the scheme of shallow-buried tunnel adjacent to mountain gradually replaces the deep road cut, which has become a main structural form of the mountainside road. The surface of the tunnel zone in the tunnel adjacent to mountain are sloped, at the same time, it forms cavern during digging with shallow depth and spatial asymmetry, which is basic cavern structure for half bright and half dark cavern, and also has the coordination of technical problems in engineering safety and environment protection.

In order to solve this technical problem, a new type of tunnel structure-permeable tunnel with ribbed arch beam is appeared[1, 2], which uses a measure of anchoring slopes in advance to excavate tunnel directly for a cavern in hillside, avoiding the destruction of the surface vegetation, also, realizing the coordination of the engineering and environment. For accommodating the requirement of the construction for large span and double line with a new type of environmental protection tunnel, proposing a new structure of the permeable rib double-arch tunnel further, to solve the stability of the permeable rib double-arch tunnel with spatial structure in the shallow bias area.

\section{The technical feature of the permeable rib double-arch tunnel}

Structure Feature. The permeable rib double-arch tunnel is an embedded asymmetric structure and the major structural components involve the following aspects: the ground beam of the vault, ribbed arch beam, the spread foundation in arch springing, the lining structure of the inside and outside, inverted arch, the anchor system of the vault and arch foot, middle pilot heading, mid-partition and lining, the block for prevention the falling rocks, temporary support, etc. The component of the structure is in figure 1. 


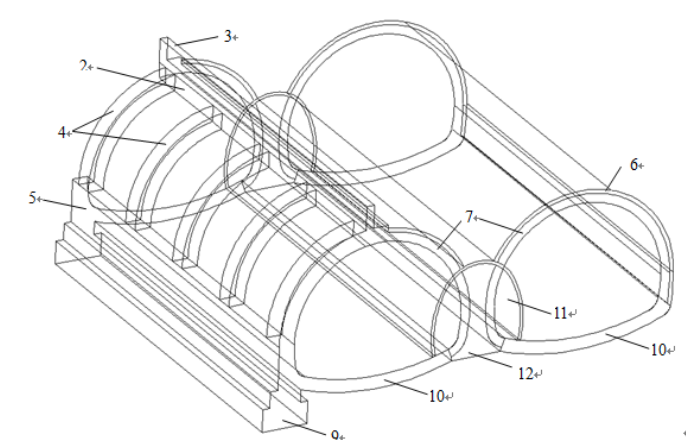

1-The anchor system of vault; 2-The ground beam of the vault; 3-The block for prevention the falling rocks; 4The ribbed arch beam; 5-The bump wall; 6-The primary lining; 7-The secondary lining; 8- The anchor system of arch foot; 9- The spread foundation; 10- The inverted arch; 11-The middle pilot heading; 12-The mid-partition

Fig. 1 The Structures of permeable rib double-arch tunnel

Technical feature. (1)The permeable rib double-arch tunnel uses double-arch structure, combining cross-mounted pipe-shed to strong support the vault slope and then excavating by steps to form the left and right caverns, avoiding the destruction of the vegetation on the hillside furthest.

(2)The Outside hole is the structure of the ribbed arch beam, having good ventilation and lighting performance; the outer hole and the inner hole are connected as a whole through the mid-partition, and balanced the inclined press load and formatted a larger double line tunnel structure, which effectively improves the traffic capacity of the tunnel with ribbed arch beam.

(3) Using integral mid-partition to effectively provide the support for the rock mass of the vault slope, avoiding the instability of surrounding rock caused by the failure of the rock mass at the arch foot and the side wall (the region of stress concentration), which reduced the safety risk of construction and excavation, at the same time, improving the applicability of the tunnel in the environmental geology.

(4)The depth-span ratio of the permeable rib double-arch tunnel is obviously reduced comparing with the single hole of the permeable rib double-arch tunnel, having the higher safety factor of the antiskid and anti-overturning property in the shallow bias area.

The difficulties of the construction technology. Transparent rib beam tunnel is mainly suitable for sloping ground surface of the mountainside area with thin layer, low surrounding rock classification and significant bias effect. As a consequence, there will be more complex construction technology problems to build a permeable rib double-arch tunnel with greater span and significant space effect.

(1)The tunnel uses more complex special-shaped structure, and the feature of the space structure is more distinct. On the other hand, it should consider fully the stability in the process of construction with the ribbed arch beam, mid-partition and lining structure under the inclined press load.

(2) The excavated surface of the tunnel is greater and the disturbed area is more extensive in the entrance to a cave and the shallow segment with loose rock mass, which has higher requirements to the support of the vault slope and surrounding rock.

(3) The tunnel's construction mechanics behavior and the space-time evolution regularities are more complex. Not only existing the problem of time-space effect in propulsion along the longitudinal, but also having a problem of interaction between left and right caves. At the same time, the construction process and condition are more complex.

Therefore, according to the structural feature and mechanics regularity of the permeable rib double-arch tunnel to explore the reasonable excavation sequence, support pattern and advance per round in construction technological parameter is the key link to ensure the success of the new tunnel.

\section{The construction technology of the permeable rib double-arch tunnel}

The geological characteristics of the engineering environment. The new tunnel is based on the project of Anhui Province, the south of the Wangjing Yangtze River Highway Bridge, located in 
the East County Xiang Yu Zhen. The origin of the stake number is K27+472 K27+636 and the total length is $164 \mathrm{~m}$, in which the section of the K27+472 K27+538 is connected with a structure of the permeable rib double-arch tunnel (ribbed lining section).

The tunnel is belonged to a landform of the low mountain denudation with narrows valleys, steep terrain, thick natural vegetation, at the same time, the ecological environment is well kept. The surrounding rock of the tunnel is mainly metasandstone, which belongs to soft rock to hard rock. The line is closely parallel to the form line, and the rate of the natural slope is about 1:1.25. If set integral subgrade, the maximum excavation height near $70 \mathrm{~m}$, which caused a very difficult management and the high project cost, also, seriously destroys the beautiful natural ecological environment, and leaves a security risk. Through economic and technical argumentation, the best way is to use the permeable rib double-arch tunnel to through.

The overall scheme of the construction and excavation. According to the main technical problems of the transparent in permeable rib double-arch tunnel, established the overall scheme of the construction and excavation. Therefore, the construction sequence of the excavation and support are as the followings: A-grouting reinforcement in vault slope, B-excavating the middle pilot heading, C-temporary support to the middle pilot heading, D-pouring the mid-partition, E-horizontal steel supports and backfilling the middle pilot heading, F-temporarily excavating the slope of the left hole, G-pouring the spread foundation, H-excavating the side pilot heading of the left hole, I-constructing the temporary support of the side pilot heading on the left hole, J-excavating the surrounding rock of the hill slope on the left hole, K-constructing the support and erecting the steel arch, L-pouring the ribbed beam of the left hole, longitudinal beam and the secondary lining, M-excavating the side pilot heading of the right hole, $\mathrm{N}$-constructing the temporary support of the side pilot heading on the right hole, O-excavating the surrounding rock of the arch on the right hole, P-constructing the support the and erecting the steel arch, Q-excavating the core soil of the right hole, S-pouring the concrete on the right hole. The next cycle construction is shown in Figure 2.

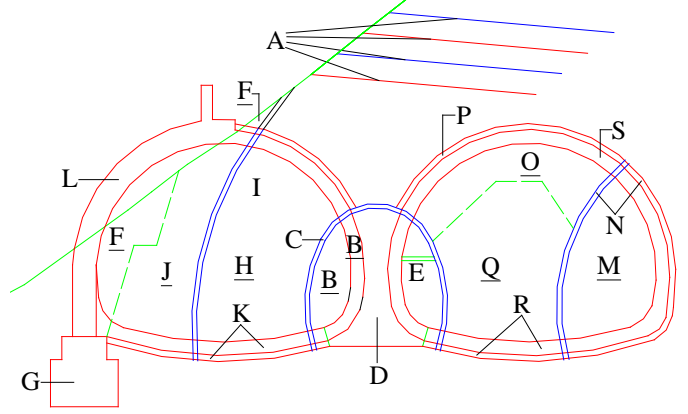

Fig. 2 The construction process of permeable rib double-arch tunnel

Protecting and reinforcing the slope. Because of the particularity of the structure of the permeable rib double-arch tunnel, the eccentric compression of the tunnel is serious, and the hole of entrance and exit are the loose media. So, in order to strengthen the surrounding rock, the vault slope should be reinforced before excavation (vault anchorage system in Figure 2), and the deviator stress level should be reduced by the control measures of the active deformation.

Before excavating the tunnel, 5 6 rows of the grouting steel tubes and 5 10 rows of the grouting small ducts are arranged on the Vault slope surface along the line. The grouting steel tubes use the hot rolled seamless steel tube with external diameter of $108 \mathrm{~mm}$, wall thickness of $6 \mathrm{~mm}$, the length of $15 \mathrm{~m}$, and the spacing is $2 \mathrm{~m} \times 2 \mathrm{~m}$, adopting the arrangement in passing quincunx [3]. The grouting pipes are inclined to 25 degrees downward at a horizontal angle, and the axis of the hole is perpendicular to the direction of the line. After the grouting pipe is injected into the surrounding rock, the cracks in the rock and the surrounding rock of the rock and the surrounding rock are combined to play a role in the vault.

The construction of the middle pilot heading and the mid-partition. The middle pilot heading is the first hole to excavate for the construction of the mid-partition with the width of $6.3 \mathrm{~m}$, the height of $6.75 \mathrm{~m}$. The ceiling of the middle pilot heading and the top of the mid-partition are close contact. The first lining layer of the middle pilot heading uses the early strength concrete of 
C25 with thickness of $20 \mathrm{~cm}$ and laid the steel mesh with $\varphi 8 \mathrm{~mm} @ 20 \times 20 \mathrm{~cm}$. The excavation face sets support bolts which use the early strength cement mortar of $\varphi 22 \mathrm{~mm} @ 60 \times 100 \mathrm{~cm}$ with the length of $3.0 \mathrm{~m}$. The support bolts exposed parts are connected with the first lining layer, forming the early support of the middle pilot heading together.

The mid-partition is the reinforced concrete structure of C30, using the style of composite curved middle-wall, and the core-wall thickness is $1.1 \mathrm{~m}$, the bottom width is $3.3 \mathrm{~m}$, the top width is $2.3 \mathrm{~m}$, the height is $6.1 \mathrm{~m}$. The two sides of the mid-partition is the secondary lining of the C30 reinforced concrete structure with the thickness of $60 \mathrm{~cm}$, and the core-wall of the mid-partition is integral concreting with the secondary lining next to the left and right hole, forming a unitary mid-partition to enhance the stability of antiskid and anti-overturning on the mid-partition during construction.

Before constructing the foundation of the middle pilot heading, needing to clean up the virtual slag and stone chips on the foundation to ensure the foundation is located on bedrock, and the over-excavation of the foundation needs to be filled with concrete. The bottom of the middle pilot heading is reinforced by anchors which are $\Phi 25$ hollow grouting anchors with length of $350 \mathrm{~cm}$, spacing of $100 \mathrm{~cm} \times 100 \mathrm{~cm}$ and is drilled into the middle pilot heading downward vertically to the horizontal plane. After pouring the middle pilot heading, and adding the temporary horizontal steel support on the right hole, backfilling the middle pilot heading with mortar rubble of M7.5, and the height of backfill is $3.5 \mathrm{~m}$ above the bottom of the middle pilot heading.

The construction of the ribbed beam and the lining on the left. The permeable rib double-arch tunnels are the shallow buried bias tunnels which effectively control the deformation of the mountain and protect the self-supporting capacity of surrounding rock, reducing the inclined press load of the middle pilot heading, also, it enhances the overall stability of structure. Constructing to the foundation of the ribbed lining on the left hole after completing the excavation and support to the middle pilot heading.

Before constructing the ribbed lining on the left hole, it should use the large-barreled shed to pre-grouting reinforcement for ensuring the stability of the vault slope. The concrete construction steps as followings:

(1)Excavating the spread foundation and the temporary surface (Figure 2 shows F). The width of the spread foundation is $3.0 \mathrm{~m}$, and the height is $2.5 \mathrm{~m}$, which is the step shaped of a reinforced concrete structure arranged lengthways along the line, and there are some small ducts with the length of $5 \mathrm{~m}$ and $\varphi 50 \times 5 \mathrm{~mm}$ on the bottom, using the step shaped arrangement with the spacing of pipe in $1 \mathrm{~m}$ for increasing the horizontal carrying capacity of the base; In the premise of ensuring stability of the slope during excavation, minimizing the excavation on the slope, and guaranteeing the soil thickness on the right, at the same time, guaranteeing the construction safety of the side pilot heading.

(2)The excavation and temporary support to the side pilot heading on the left hole (Figure 1 shows $\mathrm{H}$ ). The temporary support uses the early strength concrete of C25 with the thickness of $20 \mathrm{~cm}$, and the steel arch uses the I-steel of I16 with the longitudinal space of $60 \mathrm{~cm}$, at the same time, setting the steel mesh of $\varphi 8 \mathrm{~mm} @ 20 \times 20 \mathrm{~cm}$.

(3)Excavating the surrounding rock of the hill slope on the left hole (Figure 1 shows J), using the bench method to construct, and excavating with the method of the machinery or weak explosion. It should be strictly controlled the excavation of the cyclic footage and supported in time, in the meantime, paying attention to measuring the deformation of the cave and ground surface.

(4)Constructing the waterproof layer on the left hole, and molding the concrete on ribbed beam of the left hole, ground beam of vault roof and the secondary lining layer of the left hole totality.

The initial ribbed beam uses the dimension of $160 \mathrm{~cm}$ (longitudinal) $\times 105 \mathrm{~cm}$ (horizontal) for the ribbed lining, and the other ribbed beams use the dimension of $120 \mathrm{~cm}$ (longitudinal) $\times 105 \mathrm{~cm}$ ground beam (horizontal), which is $6.0 \mathrm{~m}$ distant from the center distance. On the top of the ribbed setting the longitudinal beam as the structure of the actuating system, and at the bottom of the ribbed beam setting the bar spread foundation of the reinforced concrete in order to ensure the structural stability of the construction process and the post-stage operation.

The secondary lining of the tunnel arch on the left hole is the shell structure of C30 reinforced 
concrete with the Thickness of $60 \mathrm{~cm}$, and the top is connected with the longitudinal beam of the vault, at the same time, the bottom is felled on the middle pilot heading on the shoulder, forming the closed bearing structure with the tunnel invert on the bottom.

The circular excavation footage is $12 \sim 14 \mathrm{~m}$, and the spacing of the longitudinal construction is $6 \sim 8 \mathrm{~m}$. The spacing of the construction on the left and right hole is $30 \mathrm{~m}$.

In order to ensure the safety of the mid-partition to construct the temporary support (I-steel, anchor bolt spray) which is not constitute the main structure of the tunnel, but it is an indispensable link in the process of the construction. Only the fulcrum of the primary support on the main tunnel acts on the top of the spread foundation can remove the temporary support of the mid-partition to ensure the load safely transferred to the top of the spread foundation.

The excavation and support on the right hole. The construction of the mid-partition on the right hole uses the bench method, and the main hole uses the method of ring-excavation with the core soil reserved. Each loop of the excavation progress is controlled in a complete calculation of the arching spacing $[4,5]$. According to the design requirements to support the hole timely after excavating, and constructing the tunnel invert to close the loop.

When excavating the top of the right hole, using the light blasting of microseism, and controlling the interval of the blasting delay time to avoid the superposition of the vibration waves, accordingly, reducing the shock of the wall with lining. It is closed to one side of the middle pilot heading and the side wall away from the support of $1.0 \mathrm{~m}$ to reserve a protective layer, at the same time, using presplit blasting of the twice-cut to ensure the safety of the lining structure.

The temporary support of the mid-partition on the right hole uses the early strength concrete of C25 with the thickness of $20 \mathrm{~cm}$ and the steel arch uses the I-steel of I16 with the longitudinal space of $60 \mathrm{~cm}$, at the same time, setting the steel mesh of A8mm@20×20cm. The excavation face sets supporting anchor pile which is the early strength mortar anchor of B 22mm@60×100cm with the length of 3.0m [6, 7].

After excavating the surrounding rock of the arch, supporting and erecting the steel arch timely.

The primary support layer uses the early strength concrete of C25 with the thickness of $26 \mathrm{~cm}$ and the steel arch uses the I-steel of I20 with the longitudinal space of $60 \mathrm{~cm}$, at the same time, setting the steel mesh of A8mm@20×20cm.

The secondary lining on the right hole is the reinforced concrete construction of C25 with the thickness of $60 \mathrm{~cm}$, and the two ends are respectively connected with the middle pilot heading and the tunnel invert, forming the circular closed bearing structure.

Enter the next stage of the construction cycle. The circular excavation footage is $12 \sim 14 \mathrm{~m}$, and the spacing of the longitudinal construction is $6 \sim 8 \mathrm{~m}$. The spacing of the construction on the left and right hole is $30 \mathrm{~m}$.

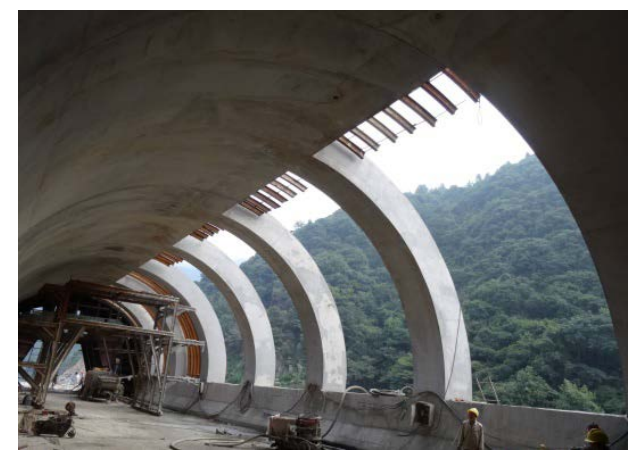

Fig. 3 The mid-partition pouring model

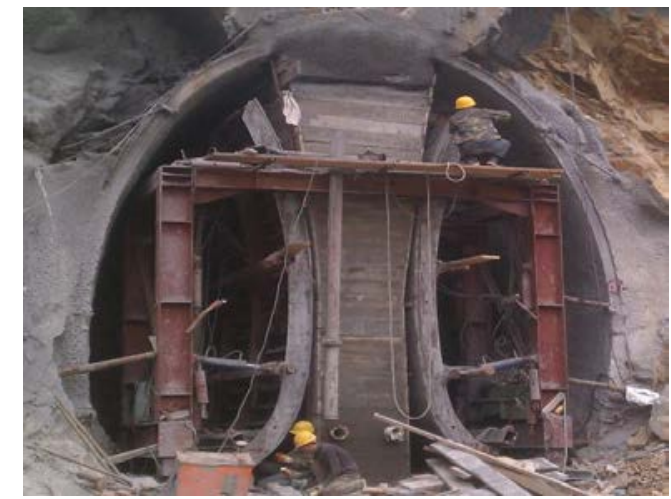

Fig. 4 The ribbed beam and bump wall in the outside tunnel

\section{The technique advantage of the construction method in the permeable rib double-arch tunnel}

To make a construction method of the permeable rib double-arch tunnel is based on the ribbed arch tunnel's construction mechanics behavior and the space-time evolution regularities. The main technical advantages are embodied in the following aspects: 
(1)The grouting steel tube of the anchor system on the vault slope is extended to the reinforced area of the bolt anchorage system on the cave, which is formed a continuous strengthen system, effectively reducing the deformation of the rock deformation of the tensile region on the vault slope, at the same time, reducing the stress level of the tunnel structure. So, it is better ensured the stability of the rock on the vault slope and surrounding rock of the tunnel.

(2)The permeable rib double-arch tunnel is an embedded asymmetric structure and the excavation process is divided into several steps. First is the excavation of the middle pilot heading and the next is the unitary mid-partition. The mid-partition and the secondary lining next to the inside and outside hole are integral concreting in advance, which has a higher bending rigidity comparing to the traditional mid-partition of 'layer cake' that is divided into three parts and there has a better sliding resistance and overturning resistance, especially in the bias section which is effectively increased the stability of the mid-partition during constructing.

(3)The excavation scheme of the construction is first in the left hole and next in the right hole. After excavating the middle pilot heading and constructing the mid-partition, excavating the left hole firstly and constructing the tunnel structure of the ribbed arch beam and so on. Forming a stable support system and then excavating the right hole. Finally, construct the tunnel structure of the right hole. The total construction scheme well assures the stability of constructing the special-shaped double-arch tunnel in bias section.

(4) The ribbed arch beam of the left hole is integral concreting with the carling of the vault and the bump wall, forming a ring bearing force system. Not only ensure the integral rigidity of the tunnel structures, but also effectively balance the inclined press load and improve the stability of the tunnel structure for a long time.

(5) Using the anchor system of the vault and arch springing to reinforce the slope body of the influence region of the excavation and reduce the deformation of the slope rock mass, thereby decreasing the stress level of the tunnel structure.

\section{Conclusion}

The construction procedure, supporting system and the construction control parameters of the permeable rib double-arch tunnel are adapted to the ribbed arch tunnel's construction mechanics behavior and the space-time evolution regularities, and effectively ensured the safety of the construction process and the stability of the tunnel structure, which provides an applicable construction technology for promoting and applying this new type of environmental protection tunnel.

Depending on successful completion of the south of the Wangjing Yangtze River Highway Bridge project, which are verified and tested the feasibility and effectiveness of construction method.

\section{References}

[1] Wang Xiangqiu, Yang Linde, Gao Wenhua. The Dynamic Construction Monitoring of Bias Tunnel on the Highway and Finite Element Simulation [J]. Chinese Journal of Rock Mechanics and Engineering, 2005, (02): 284-289.

[2] Yu Fei, Chen Shanxiong, Chen Xiuhe, etc. Structural Analysis of Permeable Tunnel With Ribbed Arch Beam [J]. Chinese Journal of Rock Mechanics and Engineering, 2009, (10): 2039-2047.

[3] Compile Group of Industry standard of the people's Republic of China. JTG F60-2009 Technical Specifications for Construction of Highway Tunnel [S]. Beijing: China Communication Press, 2009.

[4] Sun Cengwei, Chen Hao, Chen Shumao. Construction Technology of Shallow Excavation Tunnel on Taojin Station of Guangzhou Metro Line 4 [J]. Construction Technology, 2013, (08): 
57-59.

[5] Li Zhixing, Zhang Yuhua, Shi Xiaoming. Excavation Technology of Shallow-buried Tunnel Adjacent to Mountain [J]. Construction Technology, 2011, (03): 60-62.

[6] Ma Yanchun. Construction Technology of Super-shallow Buried Multi-arch Tunnel on the Highway Underneath Pass the 104 National Highway [J]. Architecture Technology, 2011, (08): 754-757.

[7] Li Xuyang. Construction Technology of Shallow Bias Tunnel [J]. Highway, 2014, 59(9): 165-167. 